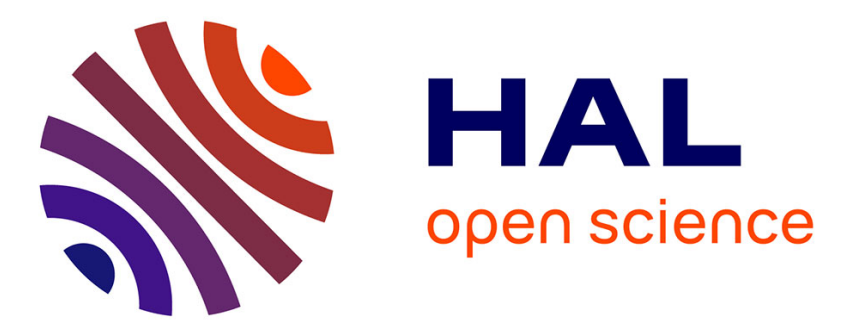

\title{
Croissance et engraissement de faisandeaux élevés en volière ou en claustration
}

\author{
F.H. Ricard, M.J. Petitjean, J.M. Melin, G. Marche, G. Malinlau
}

\section{To cite this version:}

F.H. Ricard, M.J. Petitjean, J.M. Melin, G. Marche, G. Malinlau. Croissance et engraissement de faisandeaux élevés en volière ou en claustration. Productions Animales, 1991, 4 (2), pp.117-121. hal-00895930

\section{HAL Id: hal-00895930 \\ https://hal.science/hal-00895930}

Submitted on 1 Jan 1991

HAL is a multi-disciplinary open access archive for the deposit and dissemination of scientific research documents, whether they are published or not. The documents may come from teaching and research institutions in France or abroad, or from public or private research centers.
L'archive ouverte pluridisciplinaire HAL, est destinée au dépôt et à la diffusion de documents scientifiques de niveau recherche, publiés ou non, émanant des établissements d'enseignement et de recherche français ou étrangers, des laboratoires publics ou privés. 
INRA Prod. Anim., $1991,4(2), 117-121$
F.H. RICARD (1), M.J. PETITJEAN ${ }^{(2)}$,

J.M. MELIN ${ }^{(1)}$, G. MARCHE ${ }^{(1)}$,

G. MALINEAU ${ }^{(2)}$

(1) INRA, Station de Recherches Avicoles 37380 Nouzilly

(2) INRA Domaine Pluridisciplinaire du Magneraud

17700 Surgères
Croissance et engraissement de faisandeaux élevés en volière ou en claustration

\section{L'élevage du faisan, Phasianus colchicus, s'est beaucoup développé}

en France ces dernières années en raison de l'importance que prend la chasse comme activité de loisir et de la raréfaction du gibier naturel. Il s'agit de produire de façon aussi économique que possible un oiseau de tir de qualité qui sera élevé en volière, puis laché au moment de la chasse. Pour satisfaire une demande grandissante d'oiseaux de type gibier, on peut aussi imaginer l'élevage d'un faisan de chair en claustration complète, comme le poulet, vendu dans les circuits traditionnels de distribution.

Il était normal que l'INRA s'intéresse à ce double aspect de l'élevage du faisan. Un essai a donc été mis en place pour étudier les performances du faisandeau en fonction du mode d'élevage, volière ou claustration. Nous présentons ici les résultats concernant la croissance jusqu'à 22 semaines d'âge, l'indice de consommation et l'état d'engraissement observés au cours de cette expérimentation.

\section{Conditions expérimentales}

L'expérience a porté sur les 2 sexes d'une même population expérimentale, la souche INRA du Magneraud dont les caractéristiques ont été présentées par Petitjean et al (1986). Au total, 724 faisandeaux ont été obtenus au Magneraud par insémination artificielle en juin 1989. La moitié des animaux a été élevée au

\section{Résumé}

Des faisandeaux nés le même jour et issus de la même population parentale ont été élevés soit en volière soit en claustration complète jusqu'à l'âge de 22 semaines.

Après l'âge de 10 semaines les faisandeaux élevés en volière ont une croissance plus rapide que ceux élevés en claustration, un meilleur indice de consommation apparent et un plus fort développement des dépôts gras abdominaux. Les analyses de covariance montrent que ces différences d'engraissement s'expliquent par les variations du poids vif. Les dépôts gras augmentent fortement avec l'âge et présentent une forte corrélation avec le poids vif.

Un bon compromis pour obtenir une carcasse légère et maigre semble se situer entre '14 et 17 semaines d'âge.
Domaine du Magneraud, en poulailler fermé jusqu'à l'âge de 6 semaines, dans 2 volières contigües ensuite (sexes mélangés). Les volières avaient été ensemencées d'une flore complexe, de type culture à gibier, et la densité au démarrage était de $3 \mathrm{~m}^{2}$ par sujet. L'autre moitié a été envoyée à la Station de Recherches Avicoles de Nouzilly pour y être élevée dans 2 cases d'un poulailler sans fenêtre habituellement utilisé pour le poulet, avec une densité au départ de 5 sujets (sexes mélangés) par $\mathrm{m}^{2}$. En cours d'élevage, les densités ont diminué par suite de la mortalité (faible après 6 semaines d'âge) et surtout des prélèvements pour abattage. En fin d'expérience (22 semaines) les densités étaient de 1 sujet pour $10 \mathrm{~m}^{2}$ de volière et de 1,4 sujet par $\mathrm{m}^{2}$ de poulailler. Dans les deux modes d'élevage, les animaux ont reçu un même aliment dont les caractéristiques figurent au tableau 1. Ces caractéristiques tenaient compte des travaux antérieurs effectués à l'INRA, en particulier par Melin et Larbier (1988).

Tous les animaux ont été pesés, le matin à jeun, aux âges de $6,10,14,17$ et 22 semaines. 
Tableau 1. Caractéristiques mesurées des aliments utilisés.

\begin{tabular}{|lccc|}
\hline & $\begin{array}{c}\mathbf{1}^{\text {er }} \text { âge } \\
\mathbf{0}-\mathbf{2} \text { sem. }\end{array}$ & $\begin{array}{c}\mathbf{2}^{\mathrm{e}} \text { âge } \\
\mathbf{3} \text { - } \mathbf{6} \text { sem. }\end{array}$ & $\begin{array}{c}\text { croissance } \\
\mathbf{7}-\mathbf{2 2} \text { sem. }\end{array}$ \\
\hline Energie métabolisable (kcal/kg) & 2870 & 2900 & 2920 \\
Matières azotées totales (\%) & 28,8 & 24,0 & 21,4 \\
Lysine (\%) & 1,70 & 1,28 & 1,13 \\
Méthionine + Cystine (\%) & 1,27 & 1,00 & $\mathbf{0 , 8 4}$ \\
Calcium (\%) & 1,15 & 1,15 & $\mathbf{1 , 1 5}$ \\
Phosphate disponible (\%) & $\mathbf{0 , 4 0}$ & $\mathbf{0 , 4 0}$ & $\mathbf{0 , 4 0}$ \\
Anticoccidien & coyden & coyden & coyden \\
Anti syngamose (vers rouges) & 0 & Cofamix FBZ 480 \\
Présentation & miettes & Granulés 2,5 mm \\
\hline
\end{tabular}

Tableau 2. Poids vif $(g)$ des seuls animaux vivants à l'âge de 17 semaines.

\begin{tabular}{|c|c|c|c|c|c|}
\hline \multirow{2}{*}{$\begin{array}{c}\text { Age } \\
\text { (semaines) }\end{array}$} & \multicolumn{2}{|c|}{ Volière } & \multicolumn{2}{|c|}{ Claustration } & \multirow{2}{*}{$\begin{array}{l}\text { Ecart-type } \\
\text { intra-groupe }\end{array}$} \\
\hline & $O(\mathbf{N}=93)$ & 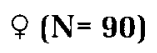 & $(\mathbf{N}=\mathbf{1 0 0})$ & $\wp(\mathbf{N}=\mathbf{9 0})$ & \\
\hline 6 & $429 \mathrm{a}$ & $365 \mathrm{~b}$ & $420 \mathrm{a}$ & $359 \mathrm{~b}$ & 37,1 \\
\hline 10 & $909 \mathrm{~b}$ & $727 \mathrm{c}$ & 943 a & $746 \mathrm{c}$ & 66,2 \\
\hline 14 & $1346 \mathrm{a}$ & 995 c & $1268 \mathrm{~b}$ & $928 \mathrm{~d}$ & 76,9 \\
\hline 17 & $1475 \mathrm{a}$ & $1085 \mathrm{c}$ & $1371 \mathrm{~b}$ & $988 \mathrm{~d}$ & 86,8 \\
\hline
\end{tabular}

a, b, c, d: pour une même ligne, 2 moyennes suivies de la même lettre ne sont pas significativement différentes $(\mathrm{P}>0,05)$.

A chacun de ces stades, la quantité d'aliment consommé a été déterminée et un échantillon de faisandeaux de chaque sexe a été abattu dans l'atelier expérimental de la Station de Recherches Avicoles. Sur les animaux abattus on a prélevé les dépôts gras abdominaux en vue d'estimer l'état d'engraissement, comme cela se fait habituellement chez le poulet (Ricard 1983).

\section{Résultats}

Chaque mode d'élevage était représenté par 2 lots d'animaux, mais pour chaque groupe âge $x$ sexe les différences n'étaient pas significatives, ce qui nous a permis de regrouper les 2 lots.

Figure 1. Croissance des faisandeaux selon le sexe et le mode d'élevage.

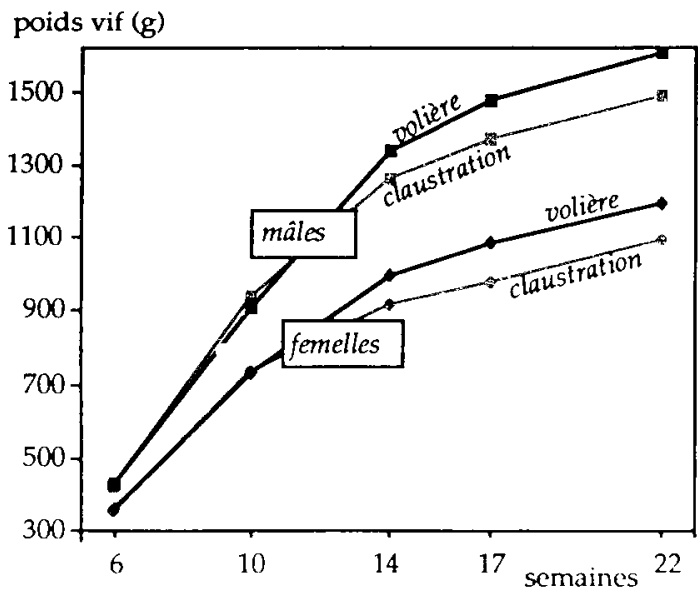

Pour tous les animaux présents, l'évolution des poids vifs est illustrée par la figure 1 . A chaque âge les effets sexe et mode d'élevage étaient significatifs, mais pas l'interaction sexe $x$ mode d'élevage. Des chiffres plus précis sont indiqués dans le tableau 2 qui correspond aux seuls animaux vivants à l'âge de 17 semaines. A l'âge de 22 semaines, les animaux restants pesaient en moyenne $1611 \mathrm{~g}$ et $1193 \mathrm{~g}$ en volière, respectivement pour les mâles et les femelles. Les valeurs correspondantes étaient de $1488 \mathrm{~g}$ et $1106 \mathrm{~g}$ pour les animaux élevés en claustration.

Ces résultats montrent que le dimorphisme sexuel du poids vif du faisan est comparable à ce qu'on observe chez le poulet et qu'il augmente avec l'âge : le poids moyen des femelles représentait $84 \%$ de celui des mâles à l'âge de 6 semaines, $79 \%$ à 10 semaines et $74 \%$ de 14 à 22 semaines. Les animaux élevés en volière étaient plus lourds que ceux élevés en claustration, sauf à l'âge de 10 semaines. Ce résultat peut s'interpréter comme une conséquence des difficultés que les animaux doivent surmonter au moment de la sortie en volière, effectuée après la pesée de 6 semaines pour l'expérimentation réalisée au Domaine du Magneraud. Par ailleurs, on peut observer que les courbes de croissance s'infléchissent très nettement après l'âge de 14 semaines. Mais le poids adulte n'était pas encore atteint à l'âge de 22 semaines. Ainsi Petitjean et al (1986) signalent des poids moyens de $1834 \mathrm{~g}$ et $1347 \mathrm{~g}$ respectivement pour des mâles et des femelles de la même souche âgés de 60 semaines.

Les résultats des mesures d'indice de consommation sont rassemblés dans le tableau 3. Les valeurs ont été obtenues sexes 
mélangés et nous n'avions que 2 répétitions par mode d'élevage. Les données ne permettent donc pas une analyse statistique précise. Elles indiquent toutefois que l'élevage en volière donne de bien meilleurs résultats que l'élevage en claustration. Jusqu'à l'âge de 6 semaines on peut penser que le gaspillage était plus important dans les poussinières de Nouzilly que dans le bâtiment du Magneraud, mais nous n'avons pû tester cette hypothèse. Après 6 semaines, les faisans élevés en volière avaient à leur disposition de la verdure et différents types de graines ce qui peut expliquer une consommation moindre de l'aliment complet, seul compté dans le calcul de l'indice de consommation. La différence est importante : à 17 ou à 22 semaines il faut $1 \mathrm{~kg}$ d'aliment complet de plus en claustration pour obtenir le même $\mathrm{kg}$ de gain de poids vif.

L'état d'engraissement a été estimé par le pourcentage des dépôts gras abdominaux rapporté au poids vif, sur un échantillon de 18 à 41 sujets par groupe sexe $\mathrm{x}$ âge $\mathrm{x}$ mode d'élevage. Les résultats sont regroupés sous forme de courbes dans la figure 2. A l'âge de 6 semaines, le poids des dépôts gras abdominaux était pratiquement nul pour la majorité des animaux. Les moyennes ne sont donc pas indiquées sur la figure 2. A l'âge de 10 semaines quelques individus présentent encore un très faible déveIoppement du gras abdominal, ce qui entraîne un coefficient de variation très élevé (80 à $110 \%$ selon les groupes). Par la suite, ce caractère reste très variable avec un coefficient de variation qui dépasse toujours $40 \%$, supérieur à ce qu'on observe habituellement chez le jeune poulet.

L'analyse statistique des résultats d'engraissement montre que les interactions sexe $x$ type d'élevage ne sont pas significatives $(P>0,05)$. A chaque âge, les effets sexe et type d'élevage sont hautement significatifs $(\mathrm{P}<0,01)$. Les mâles sont nettement plus maigres que les femelles (pourcentage de gras abdominal environ deux fois plus faible). Les animaux élevés en volière sont toujours plus gras que ceux élevés en claustration. L'effet de l'âge est très important, aussi bien chez les mâles que chez les femelles, quel que soit le mode d'élevage. En moyenne, le pourcentage gras abdominal/ poids vif double entre 10 et 14 semaines, double encore entre 14 et 17 semaines et double également entre 17 et 22 semaines. A ce dernier stade, on obtient des valeurs $(1,6 \%$ pour les mâles, $2,6 \%$ chez les femelles) qui se rapprochent de ce qu'on peut observer chez de

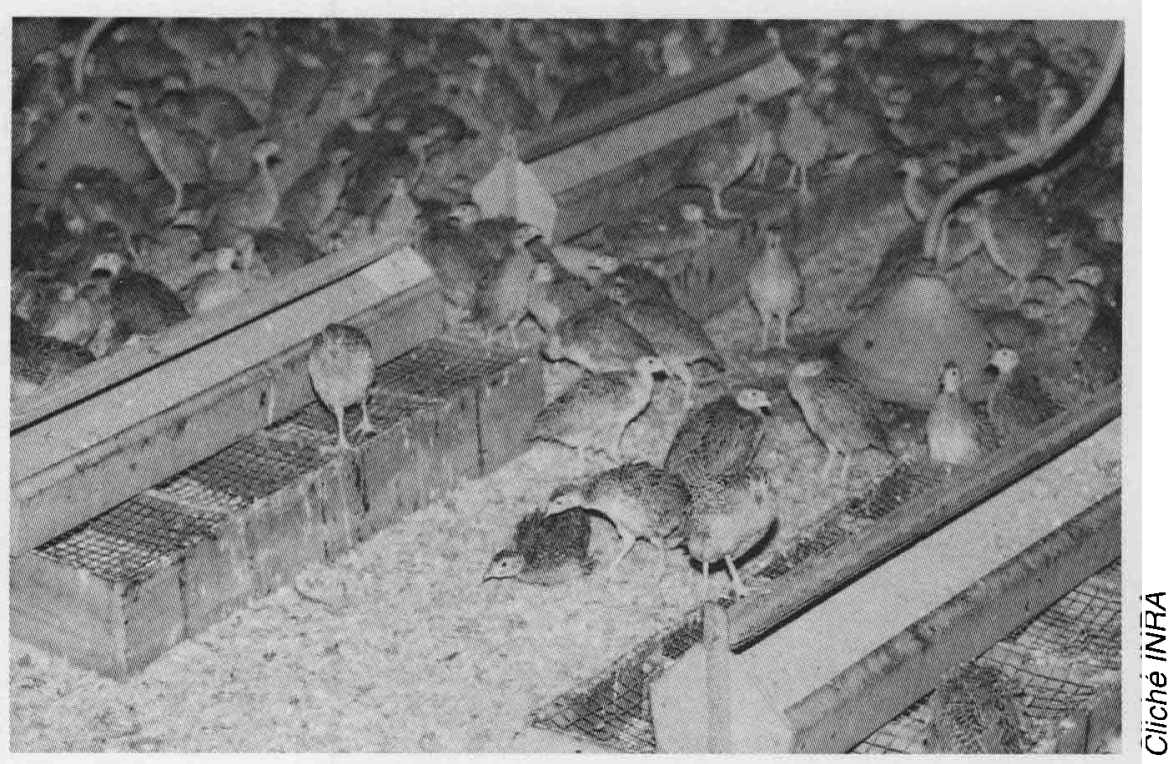

jeunes poulets âgés de 6 semaines (Ricard et Petitjean 1989).

Pour obtenir des résultats plus précis, les relations entre le poids des dépôts gras abdominaux ( $\mathrm{y}$ en grammes) et le poids vif ( $\mathrm{x}$ en grammes) ont été étudiés séparément pour chacun des deux modes d'élevage, par sexe et par âge d'abattage, par les techniques de régression et d'analyse de covariance à poids vif constant (Dagnelie 1975). Les résultats sont regroupés

Figure 2. Evolution de l'état d'engraissement selon le sexe et le mode d'élevage.

gras abdominal en $\%$ du poids vif

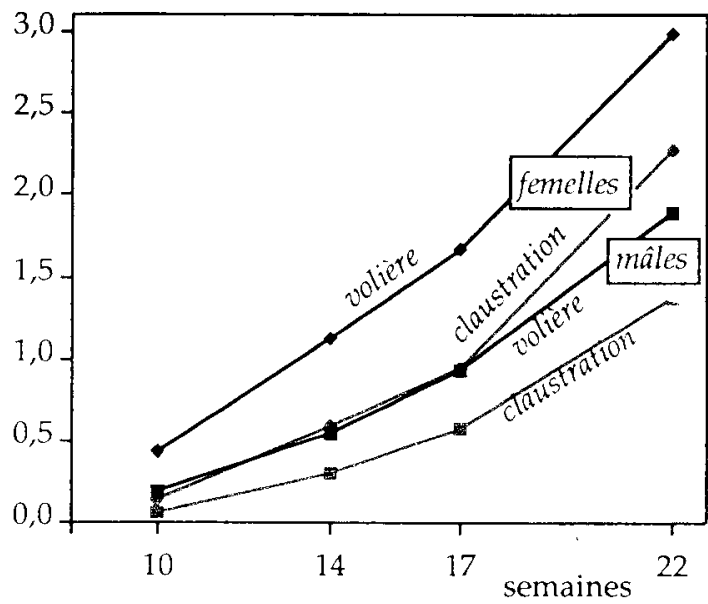

Les faisandeaux élevés en claustration complète ont un indice de consommation plus élevé que ceux élevés en volière : à 17 semaines, il faut $1 \mathrm{~kg}$ supplémèntaire d'aliment pour obtenir la même croissance.

Tableau 3. Indice de consommation (sexes mélangés) entre l'éclosion et l'âge des pesées. L'indice est exprimé en g d'aliment consommé par g de gain de poids vif. Chaque point est la movenne de deux déterminations.

\begin{tabular}{|c|c|c|}
\hline Période & Volière & Claustration \\
\hline $0-6$ semaines & 1,85 & 2,36 \\
$0-10$ semaines & 2,44 & 3,17 \\
$0-14$ semaines & 3,05 & 3,97 \\
$0-17$ semaines & 3,43 & 4,50 \\
$0-22$ semaines & 3,91 & 4,91 \\
\hline
\end{tabular}


Tableau 4. Corrélations phénotypiques et régressions du poids des dépôts gras abdominaux (y) sur le poids vif ( $x$ ), $x$ et $y$ étant exprimés en grammes.

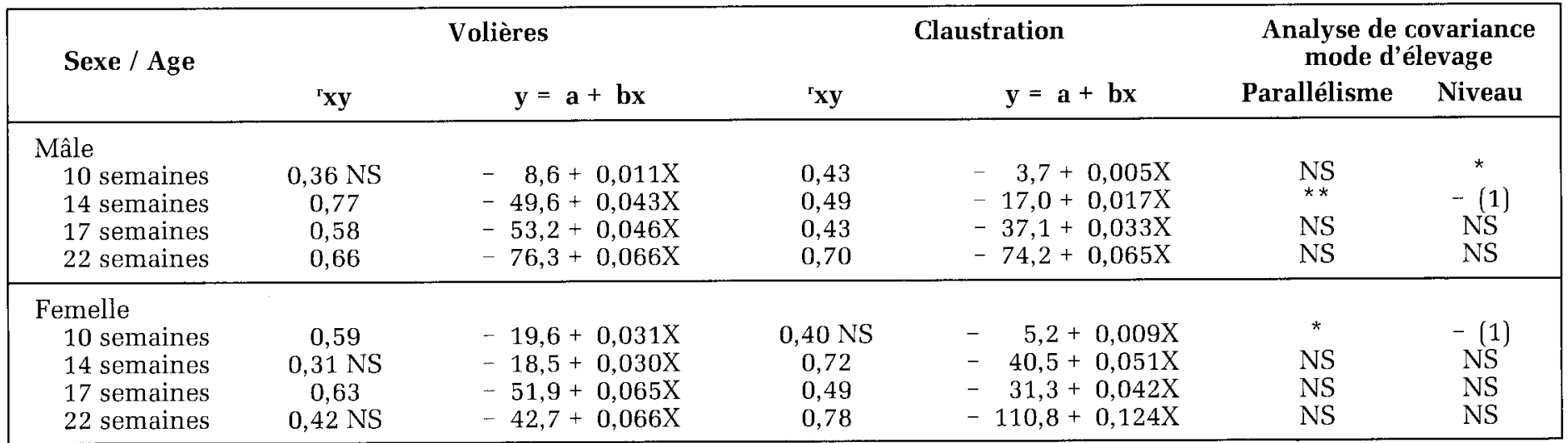

$\mathrm{NS}=\mathrm{P}>0,05 ;{ }^{*}=\mathrm{P}<0,05 ;{ }^{*}=\mathrm{P}<0,01$

(1) Test de niveau non valable car les droites de régression sont statistiquement non parallèles.

L'élevage en volière permet d'obtenir un faisan plus lourd et plus gras. dans le tableau 4. Les corrélations phénotypiques entre poids vif et poids du gras abdominal sont relativement élevées $[0,3$ à 0,8 selon les groupes) et presque toujours significatives. Les comparaisons entre modes d'élevage par analyse de covariance montrent que, dans la majorité des cas, les différences d'engraissement s'expliquent par les variations du poids vif. Les comparaisons entre âges dépendent du mode d'élevage. Pour les animaux élevés en volières, les droites de régression peuvent être considérées comme parallèles et l'âge fait apparaître une différence significative du niveau d'engraissement, aussi bien pour les mâles que pour les femelles. Pours les animaux élevés en claustration les droites de régression sont significativement non parallèles, ce qui rend impossible les tests de niveau d'engraissement.

Nous avons également calculé les corrélations entre le pourcentage des dépôts gras abdominaux et le poids vif. Les valeurs observées sont plus faibles qu'avec les poids bruts. La valeur commune, obtenue après transformation «argument tangente hyperbolique » (Dagnélie 1975), est de 0,49.

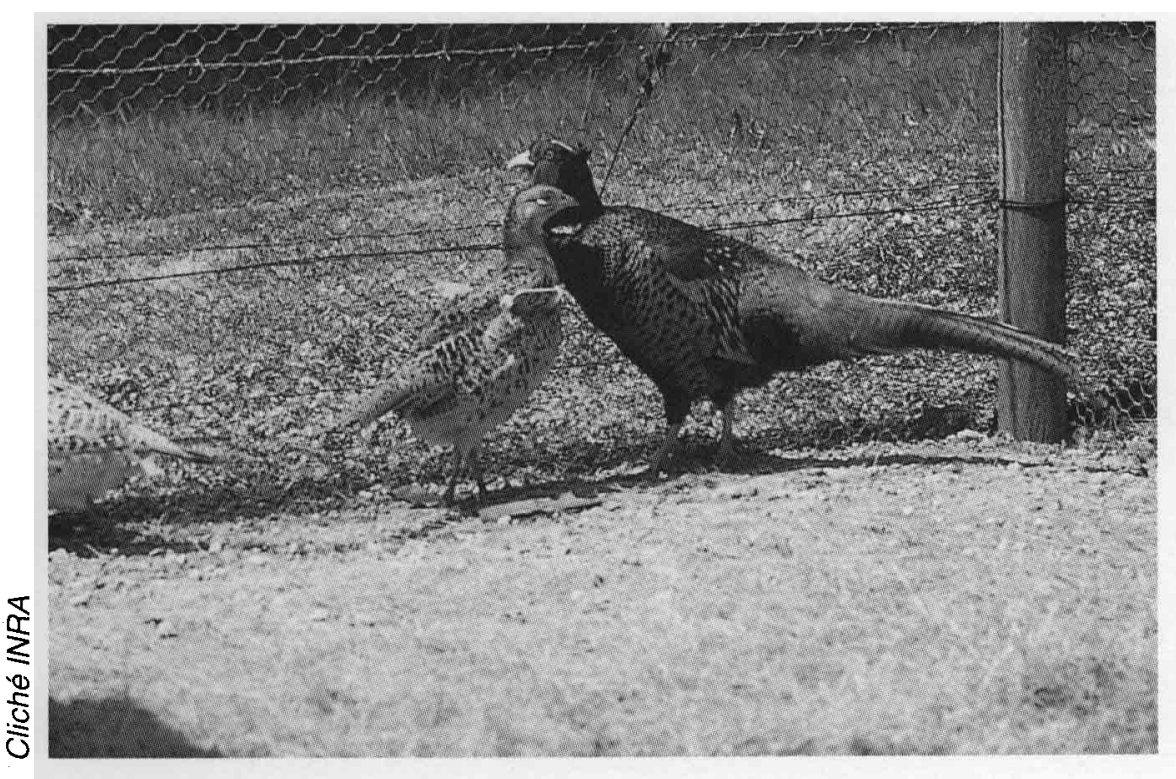

\section{Discussion et conclusion}

Les performances que nous avons obtenues sont supérieures à la plupart des résultats publiés jusqu'ici, tant pour la croissance que pour l'indice de consommation. Toutefois avec la même souche, Petitjean et al (1986) font état d'une croissance un peu meilleure. Une expérience de sélection destinée à augmenter le poids vif à 12 semaines d'âge a permis à Kassid et Coleman (1985) d'obtenir une lignée dont la croissance est légèrement supérieure à ce que nous avons obtenu. De même, Slaugh et al (1989) obtiennent une meilleure croissance sur des faisans blancs d'une souche lourde (= Junmbo White) élevés en cage, mais avec un indice de consommation moins intéressant. Cette variabilité dans les résultats montre qu'il est possible de sélectionner les performances de croissance chez le faisan, comme pour les autres espèces de volailles.

Il existe peu de données bibliographiques concernant l'état d'engraissement du faisan. Nous confirmons que la corrélation entre vitesse de croissance et engraissement est plus élevée chez le faisan que chez le poulet (Ricard et Petitjean 1989). On risque donc d'aboutir rapidement à des carcasses trop grasses si on sélectionne sur la vitesse de croissance sans tenir compte de la composition corporelle.

Si on s'intéresse à la production d'un faisandeau de chair, les résultats de la présente expérience montrent qu'un abattage à l'âge de 17 semaines permet d'obtenir un animal léger (environ 1,4 kg pour les mâles et $1 \mathrm{~kg}$ pour les femelles) et maigre (moins de $1 \%$ de gras abdominal). Si on veut améliorer le poids vif, il faudra tenir compte également de la composition corporelle.

L'élevage en volière jusqu'à l'âge de 5 mois permet d'obtenir un oiseau plus lourd et plus gras, ce qui correspond à une accumulation de réserves énergétiques. On peut penser que ce phénomène traduit une préparation pour la vie en plein air durant les rudes mois d'hiver, en attendant la reproduction naturelle du printemps suivant, ce qui se passe si les animaux sont lachés en milieu naturel 


\section{Références bibliographiques}

DAGNELIE P., 1975. Théorie et Méthodes Statistiques, Vol. 2, Les méthodes de l'inférence statistique, Presses Agronomiques, Gembloux (Belgique).

KASSID J.FH, COLEMAN T.H, 1985, Divergent selection for twelve-week body weight of Ring-necked pheasants. Iraqi J. Agric. Sci., 3, 7-19.

MELIN I.M., LABIER M., 1988. Influence du taux protidique de l'aliment de démarrage sur les performances de croissance et d'emplumement du faisan. Ann. Zootech., $37,143-150$.

PETITJEAN MJ, GUILLOT P., MALINEAU G., 1986. Maîtrise de la reproduction du faisan. C.R. $7^{\circ}$ Conf Europ. Avic. (Paris, Août 1986), LARBIER M. Ed., Vol. 1 pp. $148-152$
RICARD FH., 1983. Mesure de l'état d'engraissement chez le poulet. Variabilité d’origine génétique. in : "Qualité des viandes de volailles ». C.R. 6 ${ }^{\circ}$ Symp. Europ. Qualité viandes volailles (Ploufragan, mai 1983). Lahellec C., Ricard F.H. et Colin P. Eds., pp 49-68.

RICARD F.H., PETITJEAN M.J., 1989. Composition anatomique du faisan de tir. Comparaison avec des poulets de poids similaire. Ann. Zootech., 38, 11-18.

SLAUGH B.T., JOHNSTON N.P., FLINDERS J.T., BRAMWELL R.K., 1989. Effect of light regime on growth and feathering of jumbo white pheasants. Poultry Sci., 68 Suppl. 1, 130.

\section{Summary}

Growth rate and abdominal fat of game-type pheasants reared in aviaries or in confinement Day-old pheasants from the same parental population were reared to 22 weeks of age either in cultivated aviaries or in a standard chicken house.

Aviary reared birds were heavier from 10 weeks onwards, with better apparent feed conversion and higher abdominal fat index. Abdominal fat deposits dramatically increased with age and presented high correlation coefficients with body weight.

An age of 17 weeks appeared to be a good compromise to obtain a light body weight (1.4 kg for males, 1.0 for females) and a lean carcasse.

RICARD F.H., PETITJEAN M.J., MELIN J.M., MARCHE G., MALINEAU G., 1991. Croissance et engraissement de faisandeaux élevés en volière ou en claustration. INRA Prod. Anim., 4 (2), 117-121. 